\title{
Effectively Synchronizing 4D-STEM Detectors with Probe Movement
}

\author{
Benjamin Bammes ${ }^{1 *}$, Chenyu Zhang ${ }^{2}$, Robert Bilhorn ${ }^{1}$ and Paul M. Voyles ${ }^{2}$ \\ 1. Direct Electron, San Diego, CA USA. \\ 2. Department of Materials Science and Engineering, University of Wisconsin-Madison, Madison, WI \\ USA. \\ * Corresponding author: bbammes@directelectron.com
}

Use of a pixelated detector in scanning transmission electron microscopy (STEM) enables 4D-STEM, which is the simultaneous acquisition of both the dark-field (DF) and bright-field (BF) diffraction patterns at every STEM probe position on the specimen. By detecting the scattering angle of nearly all the primary electrons that interact with the specimen, this technique promises to provide much more information about the specimen than conventional STEM using only conventional integrating detectors [1].

One experimental challenge to using pixelated STEM detectors is their dramatically limited speed compared to conventional BF, annular dark-field (ADF), and high-angle annular dark-field (HAADF) detectors. Since the pixelated detector must record an image at each STEM probe position on the specimen, the relatively slow speed of these detectors severely limits the specimen field-of-view and resolution that can be captured in a reasonable amount of time and without deleterious effects of specimen or beam instability over long acquisition times.

Newer complementary metal-oxide semiconductor (CMOS) based detectors promise the higher frame rates (together with single-electron sensitivity) that are necessary for practical 4D-STEM data collection. However, optimizing 4D-STEM data quality requires that the detector's frame rate be carefully synchronized with the movement of the STEM probe across the specimen, so that each frame from the detector records the diffraction from one and only one STEM probe position on the specimen.

One potential obstacle to achieving synchronization is the common use by CMOS sensors of a rolling shutter [2]. In rolling shutter mode, each row from the sensor is read out sequentially from top to bottom throughout each acquisition. For simple CMOS pixel designs, this pipelined readout enables high-speed data acquisition. However, with rolling shutter readout, the timestamp of each row varies across each frame, ultimately making it impossible to synchronize the readout of the entire sensor (all rows) with any single external event. Most CCD cameras, in contrast, utilize a global shutter in which every pixel in an image corresponds to the same timestamp.

To evaluate the influence of shutter mode on the synchronization between the camera frame rate and the STEM probe movement, we performed a simple experiment where the scan generator moved STEM probe alternately between an empty specimen area and a copper grid bar. The camera's trigger delay was adjusted to maximize the difference in the average intensity of the bright-field disk for frames when the STEM probe was over an empty specimen area (where the intensity should be maximized) and when the STEM probe was over the copper grid bar (where the intensity should be minimized). We repeated experiment for a DE-16 camera operated in rolling shutter mode and in global shutter mode.

Figure 1 shows that rolling shutter acquisition mixes information from the two probe positions in every acquisition and that global shutter acquisition does not. In rolling shutter, the intensity difference between bright and dark frames is lower, and there is intensity gradient across the bright-field disk (Fig. 1a, top). These artifacts were always visible in rolling shutter data, regardless of the camera's trigger delay setting. 
Global shutter readout showed a negligible intensity gradient and better separation in intensity between the bright and dark frames (Fig. 1a, bottom), indicating that frame readout from the pixelated detector was synchronized with the movement of the STEM probe, and that each frame corresponded to exactly one STEM probe position. Fig. 1b shows line profiles from top to bottom of the images in Fig. 1(a), showing the intensity gradients in the rolling shutter data and not in the global shutter data.

We conclude that global shutter readout is beneficial for 4D-STEM data acquisition, and that rolling shutter readout runs the risk of introducing artifacts in 4D-STEM data sets.

\section{References:}

[1] H Yang, L Jones, H Ryll, M Simson, H Soltau, Y Kondo, R Sagawa, H Banba, I MacLaren and PD Nellist, J. Physics: Conference Series 644, conference 1.

[2] CK Liang, LW Chang and HH Chen, IEEE Trans. Image Proc. 17 (2008), p. 1323-1330.

[3] We thank Mark Ellisman at the University of California San Diego for use of his Titan Halo STEM to collect the data shown in Figure 1.

[4] Development of 4D STEM at UW-Madison is supported by the Wisconsin MRSEC (DMR-1720415). [5] Direct Electron acknowledges support from the Department of Energy (Office of Science, Grant DESC0018493).
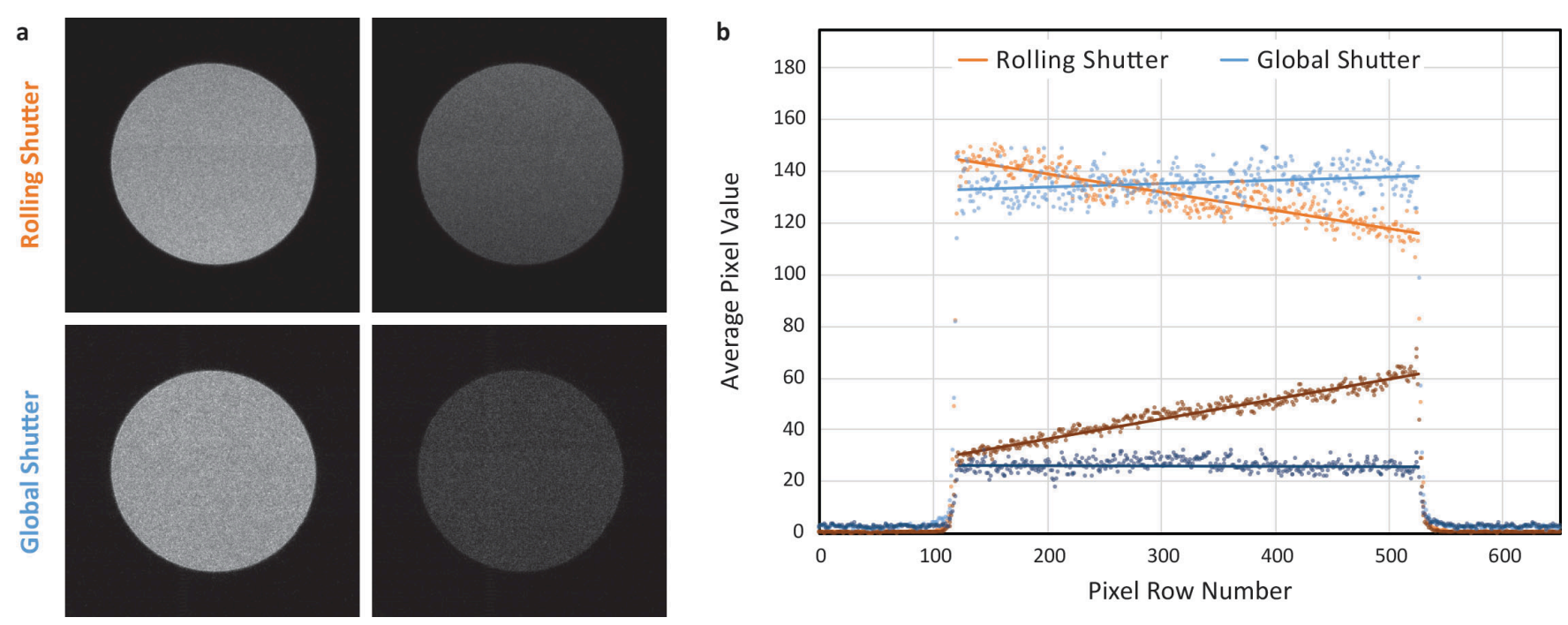

Figure 1. Comparison of rolling and global shutter. (A) Images of the bright-field disk was acquired such that the TEM beam position alternated between an empty area of the specimen and a copper grid bar in synchronization with the frame rate of the camera. The left side shows the images of the bright-field disk when the beam was over an empty specimen area, and the right side shows the images of the bright-field disk when the beam was over the copper grid bar. (B) Pixel intensity profiles across a 36-pixel wide vertical stripe in the center of each bright-field disk, corresponding to the images in A. The line of best fit over the pixels in the bright-field disk is shown on top of the actual data points. 